Proceedings of the International School and Conference on Optics and Optical Materials, ISCOM07, Belgrade, Serbia, September 3-7, 2007

\title{
Holographic Measurement of Dental Tissue Contraction and Stress, due to Postpolymerization Reaction
}

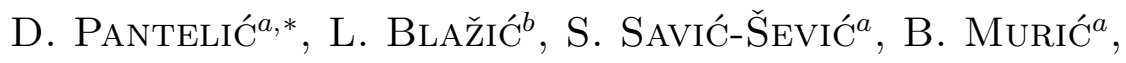 \\ D. VAsiluević ${ }^{a}$, B. PANić ${ }^{a}$ AND I. Belić ${ }^{a}$ \\ ${ }^{a}$ Institute of Physics, Pregrevica 118, 11080 Zemun, Belgrade, Serbia \\ ${ }^{b}$ University of Novi Sad, Faculty of Medicine, Dental Clinic, Novi Sad, Serbia \\ Polymerization contraction of dental photo-polymer composites induces \\ internal stress, in a tooth cavity. This stress is measured indirectly by, first, \\ holographic determination of tooth deformation, with consequent calcula- \\ tion of stress using finite element method. Real time holographic technique \\ enabled observation of dental deformation in all stages of polymerization. \\ Specially constructed blue-LED light source was used to polymerize pho- \\ topolymer composite. We have found that polymerization continues even \\ after illumination is over. Consequently, stress is increased, too.
}

PACS numbers: 42.40.Kw, 87.85.G-, 87.15.rp, 87.10.Kn

\section{Introduction}

Contraction of dental polymers during polymerization is a long standing problem in dentistry [1]. When placed inside tooth cavity, polymer contraction is transferred to surrounding hard tissues (dentine, enamel). This, in turn, induces internal stress, possibly resulting in pain, dental filling debonding or dental microcracking. It is therefore important to observe tooth deformation during dental filling placement. This makes possible to choose the most appropriate placement and polymerization techniques. The aim of this research was to investigate the postpolymerization process and additional stress build-up inside the tooth.

Human third molar with mesio-ocluso-distal (MOD) cavity were used in this study. Cavity was filled with dental composite photopolymer and polymerized using blue-light LED lamp. A real-time holography was used to measure deformation of dental tissue due to dental filling contraction.

*corresponding author; e-mail: pantelic@phy.bg.ac.yu 
Finite element method (FEM) was used to calculate stress inside a tooth, based on deformation measurement. A simplified model of a human third molar was constructed and used to calculate von Mises and principal stresses.

\section{Experimental procedure and results}

Split-beam holographic setup with liquid gate was used to in situ expose and chemically process holographic plate [2]. $532 \mathrm{~nm} \mathrm{Nd:YAG} \mathrm{laser} \mathrm{was} \mathrm{utilized}$ as a coherent light source. SLAVICH green sensitive holographic plates were used to record holographic information. Plates were developed in D76 developer and fixing step was omitted, in order to suppress emulsion shrinkage.

Human tooth was mounted in a holder using dental gypsum. A cavity (mesio-ocluso-distal) was drilled and filled with composite, using a procedure recommended by a composite manufacturer. Prepared tooth is placed in a holographic setup and a hologram is recorded and chemically processed. LED lamp is turned on for a period necessary to fully polymerize the composite.

If a tooth is observed through the hologram, interference between holographically recorded and directly reflected wave fronts can be observed. A system of interference fringes is seen on the tooth surface. Number of fringes gradually increases until the illumination is over. Interference pattern was observed and recorded by a CCD camera from the very beginning of polymerization.

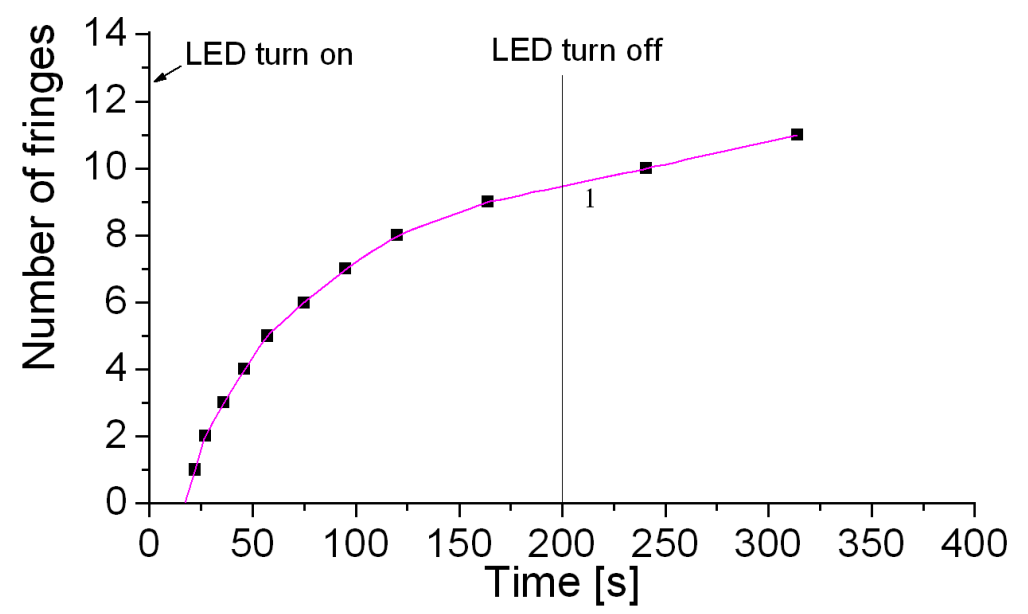

Fig. 1. Number of interference fringes observed on a tooth as a function of time.

Three phases were distinctly observed: initial stage, when the polymer is still viscous and contraction is compensated through polymer flow; contraction stage, where contraction forces gradually build up inside a tooth, and finally, postpolymerization occurs after the termination of polymer illumination and continues contraction for some time. All three phases can be easily identified in Fig. 1. We 
have found that the number of fringes as a function of time closely follows a simple exponential relation:

$$
y=A-\mathrm{e}^{-B(x-C)},
$$

where $A, B$, and $C$ are constants.

Internal dental tissue stress was calculated using finite element method applied to a mechanical tooth model (see Fig. 2, and compare it to real tooth, shown in Fig. 3). Uniform pressure was applied to cavity sides. Pressure value was chosen so that the calculated deformation of a tooth cusp was identical to experimentally measured.

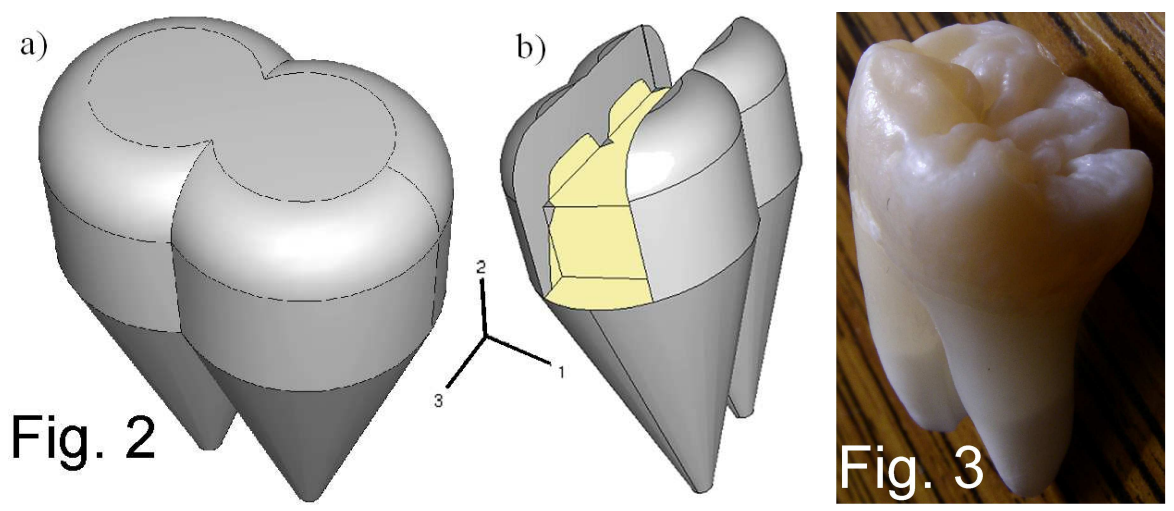

Fig. 2. (a) Intact tooth model, (b) tooth model with MOD cavity.

Fig. 3. Photograph of a human third molar (compare to Fig. 2a).

We have found that postpolymerization reaction continues for several minutes, following illumination. During that period, contraction is further increased for approximately 10\%, and maximal principal stress reaches up to $70 \mathrm{MPa}$. It should be noted that tooth may crack under pressures ranging from 50 to 150 $\mathrm{MPa}$, depending whether forces are tensile, contraction or shear.

\section{Conclusions}

Our results have shown that there is a dark, postpolymerization reaction, lasting several minutes after the illumination period has ended. During that period deformation is increased for additional $10 \%$. Consequently, internal stress is increased, too. Therefore, when analyzing various polymerization strategies, postpolymerizaion reaction should be taken into account.

\section{Acknowledgments}

This work is realized with the support of the Serbian Ministry of Science and Environmental Protection, through contract No. 141003. 


\section{References}

[1] A. Versluis, D. Tantbirojn, M.R. Pintado, R. DeLong, W.H. Douglas, Dent. Mater. 6, 554 (2004).

[2] D. Pantelić, L. Blažić, S. Šavić-Šević, B. Murić, D. Vasiljević, B. Panić, I. Belić, Opt. Express 15, 6823 (2007). 